QUADERNS DE FILOSOFIA VOL. VI NÚM. I (2019): I 5-27

eISSN: 234 I-3042 DOI: IO.7203/QFIA. 6.I.I 4822

EMILIO CÁCERES VÁZQueZ ${ }^{1}$

UNED

\title{
Intervals of quasi-decompositionality and mechanistic explanations
}

Received: 6/7/2018. Accepted: 17/12/2018

Abstract: It is commonly assumed that the concept of mechanism is a keytool for the scientific understanding of observable phenomena. However, there is no single definition of mechanism in the current philosophy of science. In fact, philosophers have developed several characterizations of what seemed to be a clear intuitive concept for scientists.

In this paper, I will analyze these philosophical conceptions of mechanism, highlighting their problematic aspects and proposing a new mechanistic approach based on the idea that the pertinent levels of organization for a mechanistic explanation can be identified with intervals of quasi-decompositionality. I argue that this approach allows us to consider that activities are directly derived from the entities' structure. Consequently, a mechanistic explanation implies an arbitrary but not capricious choice of an organizational level. According to this approach, interlevel causation is merely apparent and there is no place for emergent properties.

Keywords: mechanisms, emergent properties, quasi-decompositionality, systems.

\section{INTRODUCTION}

C rom a scientific perspective, observable phenomena are the result of the interactions of the elements underlying a given phenomenon. Thus, the luminous phase of photosynthesis is produced by a series of interactions

${ }^{1}$ Department of Logic, History and Philosophy of Science, UNED, Spain. Mail to epifumi@epifumi.com. 
among certain components of the green parts of plants. Explaining how photosynthesis takes place consists of defining, describing and locating the elements involved in that process and the relationships between them. For scientists, this is considered to describe a mechanism and, by extension, to do science. (MACHAMER et al. 2000, 2)

This claim, which could be summarized by saying that a mechanism is a set of parts that interact among themselves to give rise to a phenomenon, does not have, from a philosophical perspective, a single characterization. Instead, there are several different descriptions that attempt to capture the way scientists work. For example, (a) Machamer, Darden and Craver (hereafter MDC) affirm that "mechanisms are entities and activities organized such that they are productive of regular changes from start or set-up to finish or termination conditions" (MACHAMER et al 2000, 3); for Glennan (b) A mechanism is a set of parties whose interaction gives rise to a phenomenon, where such interaction "can be characterized by direct, invariant and changerelated generalizations" (GLENNAN 2002, S334), or its most recent minimum mechanism, in which "a mechanism for a phenomenon consists of entities (or parts) whose activities and interactions are organized so as to be responsible for the phenomenon." (GLennan 2017, 17); and (c) Bechtel and Abrahamsen argue that "A mechanism is a structure that performs a function in virtue of its components parts, component operations, and their organization". Phenomena are responsible for the smooth functioning of the mechanism. (BECHTEL and Abrahamsen 2005, 423).

However, although there is not a consensus about the definition of mechanism, there are different aspects that are included in all these approaches. In this paper, I will analyze these aspects and propose a new mechanistic approach that will be able to address all them avoiding the problematic issues of classical mechanistic accounts.

\section{The MECHANISTIC PROPOSAL}

It can be said that, in general terms, the different mechanistic proposals share the following four aspects, most of them already described in the foundational paper "Thinking about Mechanisms" (Machamer et al. 2000): First, mechanisms are formed by identifiable entities with certain stable and physically detectable characteristics that can be used to intervene in other processes, in addition to being physiologically plausible and relevant to explain the phenomenon under study (Craver 2006, 371). Second, 
In addition to entities, second, mechanisms are formed by activities (MAChamer et al. 2000), interactions (Glennan I996), or operations (BeChtel and Abrahamsen 2005). Despite being intuitively the same concept, MCD underline an identical ontological consideration between activities and entities, which although still defended by one of its main developers, Carl Craver $(2007,64,171,177,189),(2013,7)$ is also criticized by authors like Kuhlmann and Glennan (2014) and Glennan (2017, 21). Third, although there is no consensus on how causality should be understood, it is agreed that mechanisms play a causal role in the occurrence of the phenomenon. It can be argued that the causal approach to mechanicism was firstly dominated by Wesley Salmon's (Salmon 1994) and Phil Dowe's (Dowe 2000) approach to the conserved quantities, and is now in the line of the manipulability theories of causation, such as the account developed by James Woodward (2003). However, there are also other causal approaches such as that derived from Glennan's own mechanicism (GLENNAN I 996) or Craver's constitutive approach. Fourth, the organization to which the mechanistic approach refers to typically corresponds to the hierarchical structure of many of the systems studied by scientists. Each component of a mechanism can also be itself a mechanism susceptible of being broken down into further mechanisms, which introduces the idea of levels of organization into the mechanistic framework. (MACHAMER et al 2000, 13)

The idea of level of organization is one of the core notions for scientific research, since the choice of level depends on the entities, the actions that these entities carry out and the causal relationships among them. For instance, Craver $(2007,171)$ chooses the levels of mechanisms as the most valid option for carrying out an explanation, rejecting the classical view proposed by Oppenheim and Putnam $(1958,9)$ and the "refined" version of Wimsatt $(1976,253)$, that is, elementary particles, atoms, molecules, cells, multicellular living beings and social groups. A mechanism level is chosen, not only because of its entities, but also because of its activities. In other words, the election of the level or organization is function-oriented (cf. GARSON 20I3).

However, Craver's decomposition of the levels gives rise to some difficulties. As noticed above, for Craver, a single-level element can be recursively broken down into sub-elements. However, it cannot be said that the resulting hierarchies are comparable, so that the sub-levels are out of sync. Therefore, as Craver and Bechtel point out, the size of the entities involved is not important, as a virus and a human may be at the same level of mechanism and interact with each other. From this contextual definition of mechanism, they attempt to explain downward causation without top-down 
causes (Craver and Bechtel 2006, 556) and exclude from their analysis strongly emerging properties on the basis that they are emergent only with respect to a different level of characterization. However, most mechanicists advocate a mechanistic or organizational emergency in which superior properties are not directly derivable from those of their components because they are organization-dependent (CRAVER and TABERY 20I7).

Despite the usefulness and ubiquity of the mechanistic explanation, I will argue that mechanism-level characterization is responsible for the difficulties cited above. In this paper, I will show how the notion of mechanism can be complemented with a new proposal that interprets the levels of organization of the matter as intervals of quasi-decompositionality (ICD), which will allow us to derive activities from the structure of the entities involved. This implies to make an arbitrary but not capricious choice of organizational level, explaining interlevel causation as merely apparent and avoiding the idea of emergent properties.

\section{INTERVALS OF QUASI-DECOMPOSITIONALITY}

I propose to depart from the idea of nearly-decomposable systems introduced by Simon in 1962. According to this proposal, certain systems can be split according to an arbitrary value $\varepsilon$ into subsystems that are influenced only in an aggregative way. Unlike Simon's, my proposal considers the impossibility of distinguishing types of partitions according to their mutual influence, because no matter how small the influence between two partitions of a system is, it will never be null and void, meaning that the main premise in his account is never met. Even in the aggregated systems considered by Wimsatt (1997, S375), it can be found that the resulting properties assume more than mere accumulation. This can be seen by the classical example of a making a pile of sand, as not only does the mass and volume of the pile change, but the degree of compaction changes as well, therefore the density changes and properties such as fluidity appear. Therefore, I propose to eliminate the possibility of partitioning nature into decomposable elements, understood as those that are not influenced, and to consider that every material is a continuum in the purest style of (WeIss I970, 7).

From this starting-point, properly justified in Cáceres and Saborido (2017; 2018), it can be stated that, if instead of taking a single criterion of decompositionality $\varepsilon$ we take several criteria, it is possible to quasi-decompose the continuous material in the levels of organization of the matter (longitudinal intervals). In this way, one can decide on a value for $\varepsilon$ that will provide us with a 
fundamental or microscopic aproblematic ${ }^{2}$ level $\mathrm{F}$ that delimit the partitions at the most basic level. Following Simon, this value could be based on the energy that holds matter together. For example, we can consider as a fundamental level the one that delimits the stability of the atomic nucleus ciphered around $8 \mathrm{MeV}$, a sufficiently high value to consider this level as "aproblematic". With lower values, we could take the energy figures that delimit the chemical bond, especially the covalent ${ }^{3}$, so that we would have defined a second quasidecompositionality interval (ICD) that would determine the molecules. Below this energy level would be the molecules and above this the associations of molecules. However, this choice is not without problems, as there are covalent bonds with a binding energy almost equal to that of some molecular interactions ${ }^{4}$. This only underlines the fact that quasi-decompositionality is ubiquitous and that the choice of values in $\varepsilon$ is essentially heuristic. Thus, the same energetic criterion allows us to continue quasi-decomposing matter in successive mesoscopic nested levels $\left(m_{i}\right)$ in until we reach a macroscopic level $M$ that would encompass all matter. In this arbitrary but not capricious way, we can establish levels as quasi-decompositionality intervals equivalent to the classic atomic, molecular, cellular, organism, population and ecosystem levels, which partition nature into atoms, molecules, cells, organisms, populations, etc. This approach could provide the robustness that Glennan calls for when he says that "parts of the mechanisms must have a kind of robustness and reality other than their place within that mechanism. In principle, it should be possible to take the part of the mechanism out and consider its properties in another context" (GLENNAN I996, 53).

Once a criterion has been established to decompose nature, we must select the ICD of our interest according to the phenomenon under study.

\footnotetext{
${ }^{2}$ Level $\mathrm{F}$ is the level of the fundamental particles described by Physics, a level that is usually considered to be the ultimate level that constitutes the basis for ontological reductionism. The Standard Particle Model considers that there are two types of particles, leptons and quarks, that lack internal structure, so they are considered truly fundamental particles. However, the stability of the atomic nucleus is so great that it can be considered as aproblematic level when it comes to its difficult decomposition. In addition to MDC, who considered this level to be "aproblematic", Glennan $(\mathrm{I} 996,50)$ also referred to this fundamental level and has worked on the compatibility of the quantum level with the mechanistic explanations (KuHLmann and Glennan 20 I4). From Physics, Roger Penrose (I991, 374-5) clarifies the difference between the quantum and the classical level, explicitly pointing out that the difficulties of current Physics do not really pose a challenge to the reductionist approach.

${ }^{3}$ Among them would be the ionization energies, so we could take the ions as an intermediate level.

${ }^{4}$ There are tremendously disparate covalent bond energies between them, for example, the $\mathrm{E}_{\mathrm{O}-\mathrm{H}}$ is $500 \mathrm{KJ} / \mathrm{mol}$ while the $\mathrm{E}_{\mathrm{F}-\mathrm{F}}$ is $160 \mathrm{KJ} / \mathrm{mol}$. However, the energy of the hydrogen bond $\mathrm{E}_{\mathrm{F}-\mathrm{H}-\mathrm{-} \text {, }}$ a non-molecular bond, is $155 \mathrm{KJ} / \mathrm{mol}$.
} 
Take, for example, the nutrition of vegetables. We can study this phenomenon from different perspectives. We can, for example, evaluate the energy flows from the sun and how they affect a forest, how they are transformed into plant matter, how they affect animal life, the climate, etc. We can also focus the study on how the light is captured by the plants through the leaves, how the flow of water and salts ascends through the xylem and how the sap is then distributed through the phloem, how the stomas of the leaves serve as an air and water vapour exchange zone, etc. But our interest may lie in the capture of energy from photons by protein photosystems, such as tilacoid molecules, that follow a sequence of oxidations and reductions that channel this energy, and how it is used to convert carbon dioxide and water into organic matter. Ecology, plant physiology or molecular biology will be the sciences responsible for carrying out these investigations.

Once the ICDs and their elements have been defined, a specific science will describe them structurally and functionally according to its methodology. Thus, ecology will not even distinguish species or will only distinguish them in terms of flow values, plant physiology will focus on the individual organism, and molecular biology on the chloroplast. Each field of science will develop its own methodology and language according to its own purposes, taking only those inputs and outputs it considers necessary and establishing a model system that will simplify reality heuristically. In this particular example, the concept of photosynthesis itself has a meaning related to matter and energy flows for ecology, fluids in plant physiology, and electrons in molecular biology. That is, when ecologists, physiologists and molecular biologists talk about photosynthesis, they mean different things. This is amplified as we ascend in level so that the observed generalizations will be equally inaccurate although they may be explanatory and predictive.

Each of the above sciences goes one step further in simplification. For example, molecular biology treats chloroplast as if it were what Bertalanffy calls an "equifinal state" (BertalanfFy I976, 147), i.e. it does not consider concrete chloroplasts, but rather idealizations of chloroplast formed by a complex of a priori selected interacting elements. These sciences will take this partition as their object of study, considering only the component parts it deems appropriate to explain what is of its interest. Thus, ecology will consider ecosystems and individuals and their functioning, physiology will consider cells, and molecular biology will consider atoms and ions. From this, we can infer that each science, in addition to its own $\varepsilon$, uses as sub-quasi-decompositionaliy criteria $\left(\varepsilon_{\mathrm{i}}^{\mathrm{sub}}\right)$ those of the sciences located at lower levels. Consequently, the sciences that investigate mesoscopic levels consider the immediately superior levels, and in a similar way they take the criteria of quasi-decompositionality of the sciences of the superior 
levels as criteria of supra-quasi-decompositionality $\left(\varepsilon_{j}^{\text {supra }}\right)$. Thus, with the choice of the levels at which a science has secondary competence, what can be called the extended quasi-decompositionality range $\mathrm{IA}_{(\mathrm{i}, \mathrm{j})}=\left[\varepsilon_{\mathrm{i}}^{\text {sub }}, \varepsilon_{\mathrm{j}}^{\text {supra }}\right]$ is established.

This modeling, i.e. the description of a quasi-decompositionality interval with respect to the properties of that interval according to the science methodology of its level, implies an important simplification. A given level can be seen as a box to be opened by the science in charge of its study. In doing so, scientists will see that its interior is occupied by other enclosed boxes made up of lower-level systems, which are also studied by another science. Each box contains new boxes that correspond to another level and another science. This nesting of boxes would end at level $\mathrm{F}$ below and level $\mathrm{M}$ above. Therefore, it is inevitable that there will always be a loss of information that will have an important influence on the observation of regularities and therefore on the establishment of laws. The loss comes from taking as "real" values from the biases introduced when selecting the level, using a methodology, simplifying the previous interval, considering decomposability, etc. In addition, not all the variables that form part of a subsystem are used to describe them, but only those detected or considered relevant.

\section{Mechanical systems}

From this approach, only F-level entities have non-decomposable properties, while entities at all other levels will have a set of properties resulting from the simplification made when making the level choice. Therefore, an F-level entity will carry out actions based on their non-decomposable properties are ultimately due to their nature.

I label the result of the simplification made after choosing an ICD as a system-entity. This simplification involves not only the structural characterization, but also the selection of certain properties relevant to the explanation, which I call level properties. The system-entities are only arbitrarily stable, contrary to the idea of the classical entity of mechanicism. For example, following the example of photosynthesis, once the molecular level has been chosen, we can characterize RuBisCO (ribulose-1,5-bisphosphate carboxylase/ oxygenase) as the protein that carries out the first reaction of atmospheric $\mathrm{CO}_{2}$ fixation, that is, the enzyme that initiates the transformation of inorganic to organic matter. We do not characterize this protein by its structure, which is very variable in all autotrophs that have it, but by some of its functions. In particular, we do it based on its carboxylase action, which initiates the dark phase of photosynthesis, and because of its oxygenase function, opposite to the 
previous one, and which reduces the efficiency of photosynthesis (NAVARRO 2005, 5-9). Therefore, the $\mathrm{RuBisCO}$ is a modeling of a molecule that has some level properties that help us to explain the phenomenon that interests us, that is, photosynthesis. Therefore, the $\mathrm{RuBisCO}$ is a system-entity with certain level properties. Both its structure and properties are fully deductible from the lower level, despite the fact that they appear to be emergent properties.

The system-entities interact with others of the same level according to their level properties, configuring a mechanism that, seen from a higher perspective, supposes a system-entity of the next level.

In this way, we can define mechanosystem as follows:

A mechanosystem (MS) is a set of system-entities (S-E) belonging to a quasidecompositionality interval (ICD) whose organization is due exclusively to the properties (P) of its components.

$$
M S=d f(I C D, S-E, P)
$$

Where:

- the ICD is chosen according to the interest of the observer

- $S-E$ is an entity-system and, at the same time, a mechanism of a lower ICD

- $P$ are selected level properties of the previous ICD

The particles of the fundamental level describe salmonian world lines5, and when they interact with those of other particles of the same level they exchange preserved quantities. Each nexus involves a causal interaction. Thus, the Salmonian view of world lines with a non-zero value of a conserved quantity and the intersection of these forming world networks is valid only for the fundamental level. As we ascend the level and speak, for example, of a molecule of water, we see how its atoms describe lines of world so that from a higher ICD it is perceived as a single thicker line, which will intersect with others in non-punctual nodes. As we move up more levels, the simplifications made in determining the mechanosystems introduce errors that accumulate making it difficult to detect the nodes. A set of water molecules together with a set of certain mineral salts can be seen as a concrete entity-system - the sapthat, at a higher level of analysis, serves to explain the ascension of nutrients by the capillarity of the trachea of the xylem. Similarly, the property "capillarity" is only observable at this level and, although it can be explained from the properties of its component elements, it is more easily distinguished by the properties of the level from which it is analysed, such as surface tension or density. Again, it can be said that this level property seems to emerge from

${ }^{5}$ A world line is the collection of points in a spacetime (Minkowski) diagram that represents the history of an object." (Dowe 2000, 90). 
its components as a qualitative novelty, but it is actually nothing more than a phenomenon that can be explained in terms of the underlying level properties.

An interlevel observer will see that a fundamental $F_{0}$ situation will be caused by another previous $\mathrm{F}_{-1}$, its corresponding mesoscopic $\mathrm{m}_{0}$ will be caused by $\mathrm{m}_{-1}$ and the upper macroscopic $\mathrm{M}_{0}$ will be caused by a previous $\mathrm{M}_{-1}$. When zooming in, the viewer cannot encompass the entire macroscopic phenomenon so he focuses on some aspect of the lower level, losing perspective. The ecologist is interested in balances, the physiologist in flows, the molecular biologist in chemical reactions. However, interactions take place exclusively at the fundamental level and therefore causality exists only at this level. For instance, the RuBisCO does not come into contact with $\mathrm{CO}_{2}$ as a whole, but only certain atoms of certain amino acids in that protein do so, meaning that such interaction is only real at the atomic level.. This gives us an idea of the arbitrariness of the choice of ICDs, because even if we choose elements of the same level, we observe that there is no real causal interaction between them.

Hence, from this perspective the interaction between elements of different sizes proposed by Craver (Craver and BeChtel 2006, 456-7) is problematic . It is true that scientists speak this way when referring to molecular mechanisms, but making a leap of at most one level. An explanation of the catalytic reaction of $\mathrm{RuBisCO}$ and $\mathrm{CO}_{2}$ includes expressions such as "binding of $\mathrm{RuBisCO}$ and $\mathrm{CO}_{2}$ to the previously activated catalytic site", "formation of 2,3-enodiol by $\mathrm{C} 3$ deprotonation", "nucleophilic attack of $\mathrm{CO}_{2}$ in $\mathrm{C2}$ ", "hydration in $\mathrm{C3}^{3}$ " and "deprotonation at C3-O level, which initiates the breakdown of the C2-C3 bond leading to two 3-phospho-D-glycerate molecules" (NAVARro 2005, 6).

When all the levels are observed at the same time, it is intuitive to analyze diachronic aspects as if they were synchronic, avoiding the differential of elapsed time $d t$ and mistaking the causal explanation for the internal identity relationship. In this way, we run the risk of concluding that $\mathrm{F}_{-1}$ causes $\mathrm{M}_{0}$ and $\mathrm{M}_{0}$ causes $\mathrm{F}_{+1}$, generating a false sense of upward and downward causality which, in reality, this is only apparent.

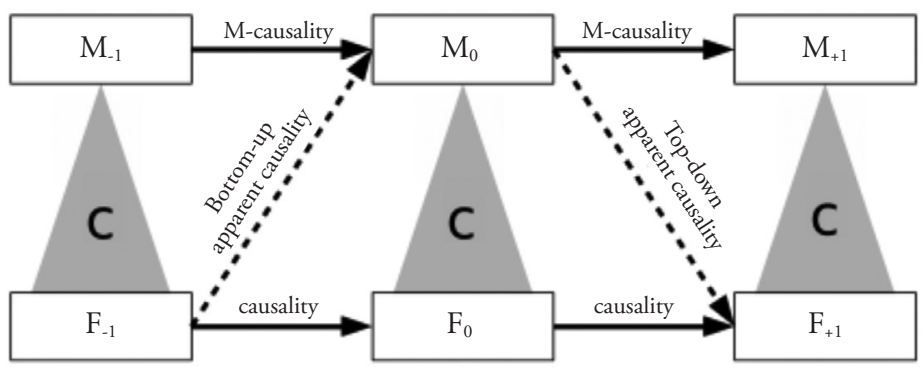

Figura 1: Apparent interlevel causality. 
Thus, the nucleophilic attack of $\mathrm{CO}_{2}$ leads to an electronic rearrangement, which seen from a molecular perspective, is the breaking of a molecule into two. If we ignore $d t$ we could believe that the nucleophilic attack is the cause of the formation of the two molecules, understanding therefore that there is an ascending causality. It is also common to express in the form of downward causation that $\mathrm{RuBis} \mathrm{CO}$ maintains its active sites, when it really depends on the sequence of the amino acid's atoms and their interaction with the dissolving water.

If we consider, as this approach does, that the world is, ontologically, a material continuum, a criterion for the choice of mechanosystems should be defined. The first thing to do would be to determine the quasidecompositionality interval. In principle, each science will choose as ICD the one that is part of its domain. Ecology, physiology and molecular biology will choose their respective ICDs. Once these levels have been established, it would be necessary to define which of the S-E's at that level are the ones that form part of the mechanism and, therefore, describe certain relevant level properties. Here we find two possible strategies: a) the elements are chosen, their properties are analyzed and the phenomena that occur are observed or b) the phenomena are chosen and the components and properties involved in their implementation are studied. Again, the decision between the two options is purely heuristic, being in general choice b) the most widely used in science. The recursive search for lower levels builds a nested set of mechanosystems. However, this top-down analysis from the phenomenon to the S-E has the disadvantage of excluding elements from the mechanism not only because of ignorance or errors, but also because of the quasidecompositionality criterion that is assumed. A complementary bottom-up study from the S-E to the phenomenon can help to better understand the functioning of the mechanism and to better characterize the properties. In the example of photosynthesis, investigating the underlying components of this plant phenomenon resulted in the discovery of the $\mathrm{RuBisCO}$ and its function. But it was also discovered that the same enzyme is the one that catalyzes photorespiration, the "opposite" phenomenon to photosynthesis. A similar case is that of the so-called multifunctional or moonlighting proteins, which are those that perform different functions depending on their environment. A striking example is that of intrinsically denatured proteins (PINE) that are capable of changing their conformation, binding different ligands and performing different functions depending on environmental conditions (Cuevas-Velázquez and Covarrubias-Robles 20i i). In order to analyze this type of protein it is very effective to examine its behavior once it has been identified and biochemically described. 


\section{Conclusions}

The consideration that mechanical systems ${ }^{6}$ are quasi-decomposable allows us to develop an approach according to which levels of organization are heuristically deduced according to the entities of the system and their specific properties. The system-entities (S-E's) are interrelated, resulting in a multitude of outcomes some of which are subject to investigation. The organization of S-E's depends exclusively on their level properties, so the consideration of emergency, as J. S. Mill already pointed out, becomes an epistemic question (Mill i 843, 267). Depending on the level of the phenomenon which we are interested in, we will carry out our research by isolating the components that are important to us, thus describing a mechanism. Thus, for example, for ecology, photosynthesis is important because it leads to the production of organic matter and the beginning of food webs and the balances of matter and energy. Its entities would therefore be the plant populations. For plant physiology, photosynthesis is important because it is the form of nutrition of individual plants and governs their life. Its entities are therefore the organs involved, such as the leaves, roots, xylem and phloem beams and, in a lower step, the stomas of the leaves, the trabeculae of the conductive vessels, the absorbent hairs, etc. For cellular and molecular biology, photosynthesis is the fixation of inorganic carbon to organic matter. Its entities are the pigments of photosystems, light photons, enzymes such as $\mathrm{RuBisCO}$ and transformed molecules. And research can also be done at more basic levels, as is the case in recent studies of quantum effects in photosynthesis (Thyrhaug et al. 2018).

In conclusion, this proposal aims to enrich the neo-mechanicist view with a systemic, reductionist and non-eliminationist approach that understands the need to address the different levels of study from their own perspective with a practical aim.

${ }^{6}$ Although some authors claim that a mechanism can be a set of processes, which have a less stable configuration (ILLARI and Williamson 20I2; GLENNAN 20I7) the approach developed here interprets mechanisms as systems in which is possible to identify quasi-decomposable intervals. 


\section{REFERENCES}

Bechtel, W. \& Abrahamsen, A. 2005, "Explanation: A mechanist alternative", Studies in History and Philosophy of Science Part C: Studies in History and Philosophy of Biological and Biomedical Sciences 36 (2): 421-41.

Bertalanffy, L. von. 1976, Teoría general de los sistemas, Fondo de Cultura Económica (ed.), Madrid.

Cáceres, E. \& Saborido, C. 2017, "Intervalos de cuasi-descomponibilidad y propiedades emergentes", Theoría 32 (1): 89-108.

CÁceres, E. \& SABorido, C. 20i8, “¿Realmente mató la bacteria al coronel?: Perspectiva sistémica, causación internivélica e intervalos de cuasidescomponibilidad en las explicaciones mecanísticas", Theoria. Revista de Teoria, Historia y Fundamentos de la Ciencia 33 (1): 129-48.

Craver, C. F. 2006, "When mechanistic models explain”, Synthese 153: 355-76.

Craver, C. F. 2007, Explaining the brain: Mechanisms and the mosaic unity of neuroscience, Oxford University Press.

Craver, C. F. 20I3, "Functions and mechanisms: A perspectivalist view", Functions: Selection and mechanisms, 133-58. Springer.

Craver, C. F. \& Bechtel, W. 2006, "Top-Down causation without top-down causes", Biology and Philosophy 22: 547-63.

Craver, C. \& Tabery, J. 20 i 7, "Mechanisms in Science", The Stanford Encyclopedia of Philosophy, E. N. Zalta (ed.), Spring 2017. Metaphysics Research Lab, Stanford University. https://plato.stanford.edu/archives/spr2017/entries/ science-mechanisms/.

Cuevas-Velázquez, C. L. \& Covarrubias-Robles, A. A. 20 i i, "Las proteínas desordenadas y su función: una nueva forma de ver la estructura de las proteínas y la respuesta de las plantas al estrés", TIP. Revista especializada en ciencias químico-biológicas 14 (2): 97-105.

Dowe, Ph. 2000, Physical Causation, Cambridge: Cambridge University Press.

Garson, J. 2013, "The Functional Sense of Mechanism", Philosophy of science 80 (3): 317-33.

Glennan, S. I996, "Mechanisms and the Nature of Causation", Erkenntnis 44: 49-71.

Glennan, S. 2002. "Rethinking mechanisms explanation". Philosophy of Science 69: S342-S353.

Glennan, S. 2017. The new mechanical philosophy. Oxford University Press.

Illari, Ph. McKay \& Williamson, J. 2012, "What is a mechanism? Thinking about mechanisms across the sciences", European Journal for Philosophy of Science 2 (1): 119-35. 
Kuhlmann, M. \& Glennan, S. 20i4, "On the relation between quantum mechanical and neo-mechanistic ontologies and explanatory strategies", European Journal for Philosophy of Science 4 (3): 337-59.

Machamer, P.; Lindley D. \& Craver, C. F. 2000, "Thinking About Mechanisms", Philosophy of Science 67 (1): 1-25.

Mill, J. S. I 843, A system of logic: ratiocinative and inductive. [8th, 1872]. London: Longmans, Green, Reader, and Dyer.

Navarro, J. V. M. 2005, "Contribución de residuos conservados de cisteína a la regulación redox del catabolismo de la Rubisco". PhD Thesis, Universitat de València.

Oppenheim, P. \& Putnam, H. I958, "Unity of science as a working hypothesis", Concepts, theories, and the mind-body problem, Minnesota Studies in the Philosophy of Science II, H. Feigl, M. Scriven \& G. Maxwell (ed.), 3-36. Minneapolis: University of Minnesota Press.

Salmon, W. C. I994, "Causality without counterfactuals", Philosophy of Science, 297-312.

Thyrhaug, E.; Tempelaar, R.; Alcocer, M.J.C.; Žídek, K.; Bína, D.; Knoester, J.; Jansen, T.L.C. \& Zigmantas, D. 20 i 8, "Identification and characterization of diverse coherences in the Fenna-Matthews-Olson complex", Nature chemistry.

Weiss, P. A. I969, "The living system: determinism stratified", Beyond Reductionism: New Perspectives in the Life Sciences, A. Koestler \& J. R. Smythies (ed.), 3-55. London: Hutchinson.

Wimsatt, W. C. I976, "Reductionism, Levels of Organization, and the Mind-Body Problem", Consciousness and the Brain: A Scientific and Philosophical Inquiry, I. Savodnik (ed.) \& W. B. Weimer (auth.), G. G. Globus, G. Maxwell. Springer US.

WimsatT, W. C. I997, "Aggregativity: Reductive Heuristics for Finding Emergence", Philosophy of Science 64 (2): S372-84.

Woodward, J. 2003, Making things happen: A theory of causal explanation, Oxford University Press. 